\title{
Fingerprint Verification applying Invariant Moments
}

\author{
J. Leon ${ }^{1}$, G Sanchez ${ }^{1}$, G. Aguilar ${ }^{1}$. L. Toscano ${ }^{1}$. H. Perez ${ }^{1}$, J. M. Ramirez ${ }^{2}$ \\ National Polytechnic Institute \\ SEPI ESIME CULHUACAN \\ Mexico City, Mexico ${ }^{1}$ \\ National Institute for Astrophysics, Optics, and Electronics \\ Electronics Department \\ Tonantzintla, Puebla, Mexico ${ }^{2}$
}

ranflash@hotmail.com,caaann@hotmail.com

\begin{abstract}
Traditional security systems use passwords or ID cards have been used to moderate access to restricted systems, but these kind of systems have a poor performance because the security can be easily breached. Based in this disadvantage, the biometrics systems have a great popularity, the mains biometrics systems are: face recognition, iris recognition, voice recognition, fingerprint recognition and sinning recognition. The fingerprint recognition is the oldest method used to recognition or verification of person. Our proposed a people recognition system with verification by invariant moments using two methodologies for the fingerprint enhancement. The goal in this work is to get a robust system in security issues. In this work a method for fingerprint verification is considered using a combination of Fast Fourier Transform (FFT) and Gabor Filters by image enhancement, both methods are first applied separately and later on an algebraic sum is done to obtain a single output. After that, a thinning algorithm is applied to get an image with the minimum thickness of 1 pixel. After this thinning algorithm, we apply an algorithm to look minutiae using a window of 3 by 3 pixels to scan the image. In this work we extract two types of minutiae, bifurcation and ending. Then, the feature vector is generated with the distance between minutiae, angle between minutiae and coordinates. In the recognition stage using the coordinates from the minutiae position on the image a comparison is done. After that, apply a verification stage using the invariant moments. With the invariant moments values a comparison is done. The comparison was done using the values obtained for the images into database and the test image for to get the output. The results obtained in this research are better when we used FFT
\end{abstract}

and Gabor filters algorithms to image enhancement than we used separately.

\section{Introduction}

The biometry or biometrics refers to the automatic identification or verification of an individual by using certain physiological or behavioral traits associated with the person. The fingerprint is the main feature that allows establishing the identity of a person. Fingerprint recognition represents the oldest method of biometric identification. Since 1897, dactylographic has been used for criminal identification. Fingerprints are fully formed at about seven months of fetus development and finger ridge configurations do not change throughout the life of an individual, except due to accidents such as bruises and cuts on the fingertips. The fingerprint has unique characteristics called minutiae, which are points where a curve track finishes, intersects or branches off. A fingerprint consists of ridges and valleys. The objective in this research is developed an algorithm that allows recognition the personal identification through their fingerprint. The proposed system in this paper, consist of two important stages. The first important stage is the image pre-processing. The second important stage is the matching. The image pre-processing consists of four steps: Acquisition, Enhancement, Cut, and Thinning. Each of these steps was evaluated with different fingerprints, some with less noise in which it was easier to work and others with information almost null, even so, our system made a good recognition. In the matching stage with the information obtained in the image pre-processing we made the minutiae detection (Local Features) and compute the distance and angle between minutiae to generate the feature vector and the feature matrix was generated. After that, in the 
recognition phase we made a comparison between the feature matrix of the store image and feature matrix of the test image. Later on, computed the invariant moments that were used in the verification phase.

\section{Pre-processing}

\subsection{Acquisition}

The acquisition of the fingerprint was made from a biometric device UareU 4000 of Digital Persona Inc. The images were captured with a resolution of 512 DPI and a size of 355 by 390 pixels in gray scale. For this work a database with 600 images of fingerprints was created, this database was obtained from 30 different people.

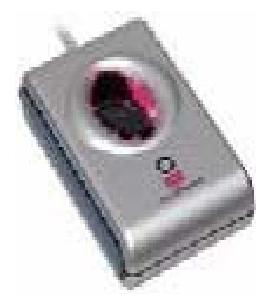

Figure 1. Optical reader.

\subsection{Enhancement}

The performance of minutiae extraction algorithms and other fingerprint recognition techniques relies heavily on the quality of the input fingerprint images.

The goal of an enhancement algorithm is to improve the clarity of the ridge structures in the recoverable regions and mark the unrecoverable regions as too noisy for further processing. The majority of the existing techniques are based on the use of contextual filters whose parameters depend on the local ridge frequency and orientation. The context information includes: ridge continuity and regularity. Due to the regularity and continuity properties of the fingerprint image occluded and corrupted regions can be recovered using the contextual information from the surrounding neighborhood [1]. In this work, we propose a combination of filters in the two domains (frequency and time) is used to improve the enhancement.

Spatial Domain Filtering: O’Gorman [2] proposed the use of contextual filters for fingerprint image enhancement for the first time. They used an anisotropic smoothening kernel whose major axis is oriented parallel to the ridges. The filter increased contrast in a direction perpendicular to the ridges while performing smoothening in the direction of the ridges. Greenberg [3] proposed the use of an anisotropic filter that is based on structure adaptive filtering by Yang [4]. Gabor filters have important signal properties such as adequately joint space frequency resolution [5]. Gabor elementary functions form a very intuitive representation of fingerprint images since they capture the periodic, yet non stationary nature of the fingerprint regions. The even symmetric Gabor has the following general form:

$$
G(x, y)=\exp \left\{-\frac{1}{2}\left[\frac{x^{2}}{\delta_{x}^{2}}+\frac{y^{2}}{\delta_{y}^{2}}\right]\right\} \cos (f x 2 \pi)
$$

Where: $f$ represents the ridge frequency, $\delta_{x}^{2}$ and $\delta_{y}^{2}$ are the spaces constants Gaussians, that determines the shape of the filter envelope and also the trade of between enhancement and spurious artifacts and $\boldsymbol{x}, \boldsymbol{y}$ these are the frequency selective and orientation selective respectively.

Fourier Domain Filtering: Sherlock and Monro [6] perform contextual filtering completely in the Fourier domain. Each image is convolved with pre computed filters of the same size as the image. However, the algorithm assumes that the ridge frequency is constant throughout the image in order to prevent having a large number of pre computed filters. Therefore the algorithm does not use the full contextual information provided by the fingerprint image. Watson [7] proposed another approach for performing enhancement completely in the Fourier domain. This is based on the 'root filtering' technique. In this approach the image is divided into overlapping blocks and in each block, the enhanced image is obtained by:

$$
\begin{gathered}
I_{e}=F F T^{-1}\left\{F(u, v)|F(u, v)|^{k}\right\} \\
F(u, v)=F F T(I(x, y))
\end{gathered}
$$

Where: $F(u, v)$ is the fats Fourier Transform of the image and $I(x, y)$ is the image.

Another advantage of this approach is that it does not require the computation of intrinsic images for its operation. This has the effect of increasing the dominant spectral components while attenuating the weak components. However, in order to preserve the phase, the enhancement also retains the original spectrum $F(u, v)$.

From the above subsections is clear that both approaches present desirable features that can be 
combined to obtain better image enhancement results. Thus, this paper proposes to use a combination of Fourier transform and Gabor filtering to carry out the image enhancement task Figure 2. As we have the two enhanced images an algebraic sum is made (Figure 2). After both enhancements, a binarization is applied to every output. This, binarization gives a lower cost computing. This step uses a threshold, the threshold is the average of the image; if the pixel value is higher than the threshold, the pixel will be white and if the pixel value is lower than the threshold, the pixel will be black.

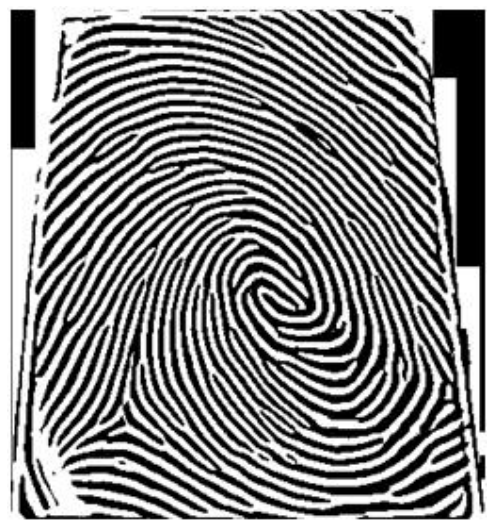

Figure 2. Fingerprint image after adding FFT and Gabor outputs.

\subsection{Cut}

After the binarization, the border in the fingerprint image is cut to avoid spurious minutiae and noise generated by the biometric scanner Figure 3 .

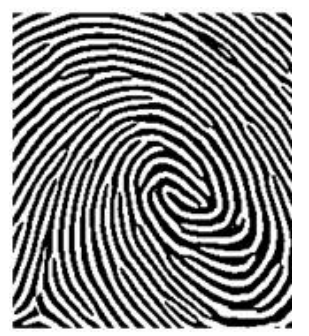

Figure 3. Cut image.

\subsection{Thinning}

The result is an image with lines of the minimum possible thickness. In order to understand better the algorithm it is necessary to know some definitions. Let us remember that after the binarization process the image consist only of 1 and 0 , where 1 means a white pixel and 0 means a black pixel.

A pixel $0(x, y)$ is internal, if its four neighbors $(x+1, y)$, $(\mathrm{x}-1, \mathrm{y}),(\mathrm{x}, \mathrm{y}+1)$ and $(\mathrm{x}, \mathrm{y}-1)$ are 0 (black pixel). The limit is defined using its 8 connections. A pixel is a pixel limit if this is not an internal pixel and at least one of its 8 neighbors is a 1. A pixel is a connection if it is eliminated in a matrix of 3 by 3 and its neighbors are disconnected.

The first step of this algorithm consists in finding the total internal pixels that exist in our image. Later, all limit pixels are eliminated, having taken care of that this is not a connection pixel. This algorithm is repeated while found internal pixels. After that, the algorithm is applied again but in this occasion with a change. This change consists in finding internal pixels only with 3 neighbor pixels and later to eliminate the limits pixels.. The last step is again the repetition of the algorithm but now, finding internal pixels with two neighbors only. Considering the elimination of an internal pixel if is not possible to eliminate some neighbors pixel. The final result after the n-th necessary repetitions is Figure 4.

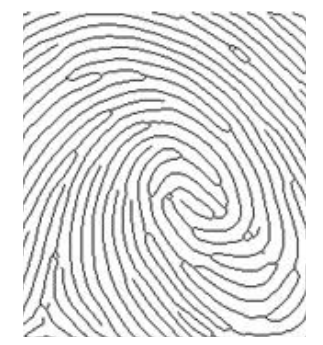

Figure 4. Thinning image.

\section{Matching}

\subsection{Minutiae Detection}

After the thinning process the image is ready so that the algorithm of detection of minutiae is applied. The algorithm consists in to calculate the number of pixels that cross to Pixel center (Pc) and it is calculated with the following equation:

$$
P C=\sum_{i=1}^{9} p_{i}
$$

Where: $p_{i=1}$ to $p_{i=8}$ are the pixels subsequent in an order that define the block of 8 neighbors of $P C$.

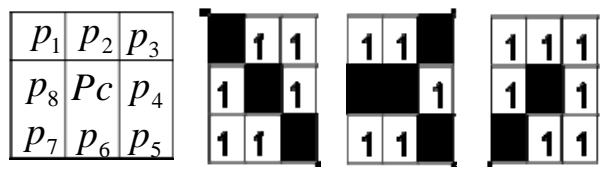


Distances and angles

a) b) c) d)

Figure 5. Window distribution and possible cases to evaluate the window.

In the Figure 5a) is observed the configuration of the used window to locate bifurcations and ending. Figure 5b) $\mathrm{Pc}=6$ means that not exist bifurcations or endings, Figure 5c) $\mathrm{Pc}_{\mathrm{c}}=5$ means that we have found a bifurcation and Figure 5d) $\mathrm{Pc}=7$ means that we are on a window with an ending, are the possible configurations that we can find. This process is made on the entire binary image applying windows of 3 by 3 Figure 6. The result of this process is a vector with the characteristic points.

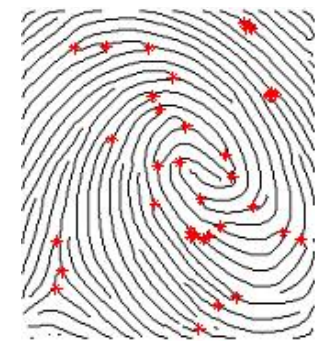

Figure 6. Final fingerprint image with the minutiae referred to as *.

\subsection{Compute the distances and angles between minutiae}

The compute of the angle is done through the stroke of a triangle between the coordinates regarding the minutiae that will make the assessment. This process is a point against all and so on for each minutia. The distance is carried out using the same triangle mentioned previously, but now we computed the hypotenuse with the Pythagoras theorem Figure 7.

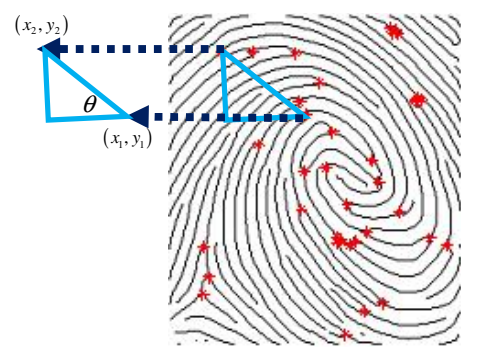

Figure 7. Triangle made to compute de distances and angles between minutiae.

$$
\begin{gathered}
D=\sqrt{\left(x_{1}-x_{2}\right)^{2}\left(y_{1}-y_{2}\right)^{2}} \\
\theta=\tan ^{-1} \frac{y_{1}-y_{2}}{x_{1}-x_{2}}
\end{gathered}
$$

Where: $x_{1}, x_{2}$ are the coordinates in the $x$ axis and $y_{1}, y_{1}$ are the coordinates on the $y$ axis.

A vector characteristic is generated for each minutia. This vector contains the distance, angle and coordinates of the minutiae. Using the features vector, the feature matrix was generated for the image. The vector is formed as the follows:

$$
\text { vector }=\left[D, \theta, x_{1}, y_{1}\right]
$$

Where: $D$ is the distance, $\theta$ is the angle, $x_{1}$ is the coordinate in $x$ axis and $y_{1}$ is the coordinate in $y$ axis.

\subsection{Recognition and Verification using invariant moments}

This section explained the recognition and verification performance to get an output. First, a comparison was made with three important characteristics: coordinates, distance and angles between each minutia. The reason to use three characteristics is to be able to have a small percentage of error in the comparison. Therefore, the information of the stored fingerprint is compound of four dates that consist of two coordinates of the first minutia, the distance of the following minutia and the angle. The comparison is made as the follows: The input image becomes a matrix and this matrix is compared with the matrix of our database. First, equal distances are located and are taken only the same angle. The coordinates which are different each other are eliminated; we can assure a better comparison. After several tests, was decide that the coordinates can vary in a radius of 10 pixels and that a greater threshold of 10 gives a good recognition, this is that the recognition exists only when the input image contains more than 10 equal values to the stored one in our database. If in a stored matrix exist more than 10 equal vectors to the input, the image is accepted.

In the recognition stage a high percentage of acceptances was obtained; however, for some images the values of coordinates, distances and angle between 
minutiae were same in more than a stored image. Therefore, for some tests the result showed more than an accepted image and the percentage of false acceptance was high. Due to this result, we made the verification test that consists of verifying the resulting images. With this test we eliminated the similar images and only the true image is accepted. Here, is the translation of the fingerprint image of test with respect to the fingerprint image that is at the database as well as its minutiae. In the translation, look for similar minutiae in fingerprint into the database and test fingerprint, in the same neighborhood. After this translation, were computed the difference between coordinates. Using these values, we translate the image and the minutiae coordinates of test image at the same point that the fingerprint into the database. After that, the Invariant Moments to Rotation and Translation are computed. The Invariant Moments are image descriptors that keep the geometry features of the image, then, after the translation, computed the seven Invariants Moments to Rotation and Translation [8].

$$
\begin{gathered}
f i_{1}=\mu_{20}+\mu_{02} \\
f i_{2}=\left(\mu_{20}-\mu_{02}\right)^{2}+4 \mu_{11}^{2} \\
f i_{3}=\left(\mu_{30}-3 \mu_{12}\right)^{2}+\left(3 \mu_{21}-\mu_{03}\right)^{2} \\
f i_{4}=\left(\mu_{30}+\mu_{12}\right)^{2}+\left(\mu_{21}+\mu_{03}\right)^{2} \\
f i_{5}=\left(\mu_{30}-3 \mu_{12}\right)\left(\mu_{30}+\mu_{12}\right) \\
{\left[\left(\mu_{30}+\mu_{12}\right)^{2}-3\left(\mu_{21}+\mu_{03}\right)^{2}\right]} \\
+\left(3 \mu_{21}-\mu_{03}\right)\left(\mu_{21}+\mu_{03}\right) \\
{\left[3\left(\mu_{30}+\mu_{12}\right)^{2}-\left(\mu_{21}+\mu_{03}\right)^{2}\right]} \\
f i_{6}=\left(\mu_{20}-\mu_{02}\right) \\
{\left[\left(\mu_{30}+\mu_{12}\right)^{2}-\left(\mu_{21}+\mu_{03}\right)^{2}\right]} \\
+4 \mu_{11}\left(\mu_{30}-\mu_{12}\right)\left(\mu_{21}+\mu_{03}\right) \\
f i_{7}=\left(3 \mu_{21}-\mu_{03}\right)\left(\mu_{30}+\mu_{12}\right) \\
{\left[\left(\mu_{30}+\mu_{12}\right)^{2}-3\left(\mu_{21}+\mu_{03}\right)^{2}\right]} \\
-\left(\mu_{30}-3 \mu_{12}\right)\left(\mu_{21}+\mu_{03}\right) \\
{\left[3\left(\mu_{30}+\mu_{12}\right)^{2}-\left(\mu_{21}+\mu_{03}\right)^{2}\right]}
\end{gathered}
$$

\section{Results}

The images for this work were captured with a resolution of 512 DPI and a size of 355 by 390 pixels in gray scale. For this work a database with 600 images of fingerprints was created, this database was obtained from 30 different people. During the translation process, the distance between the same minutiae of the fingerprint image of test and of the fingerprint image into the database is computed, with this distance translates the all minutiae and also translates the fingerprint image of test complete. Figure 8 shows the coordinates of the fingerprint image, after being translated, with respect to the coordinates of the fingerprint image into database from the same person and Figure 9 shows the coordinates of the fingerprint image, after being translated, with respect to the coordinates of the fingerprint image into database from two different persons. Although the fingerprint images were obtained from the same person, the fingerprints were not made in the same position and the same pressure. Therefore some minutiae exist in a fingerprint and in the other not and the distances in some cases are different. The following images show the graph with the invariant moments values on fingerprint images after applied translation process to fingerprint image of test.

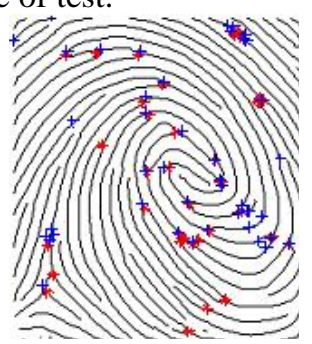

Figure 8. Fingerprint of test after translation with respect to an store image from the same person.

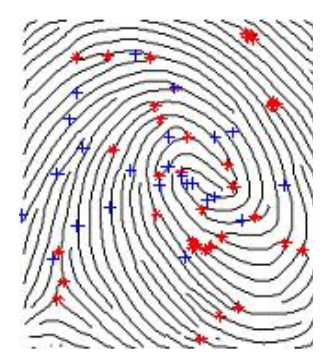

Figure 9. Fingerprint of test after translation with respect to a store image from two different persons. 


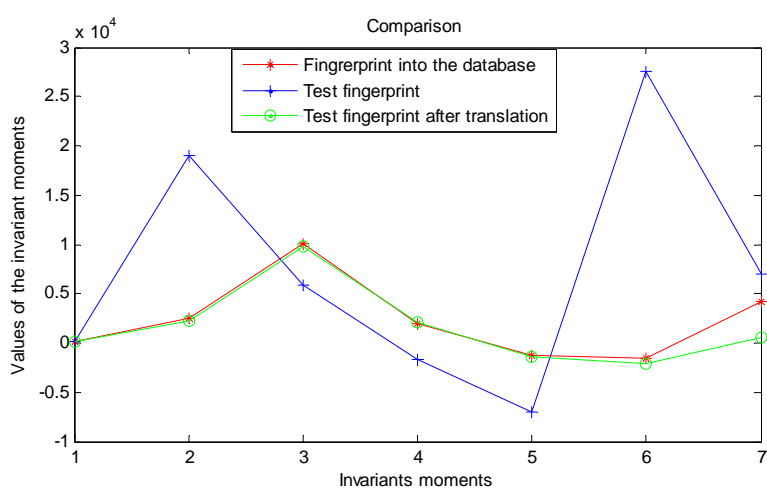

Figure 10. Graph of the invariant moments of two

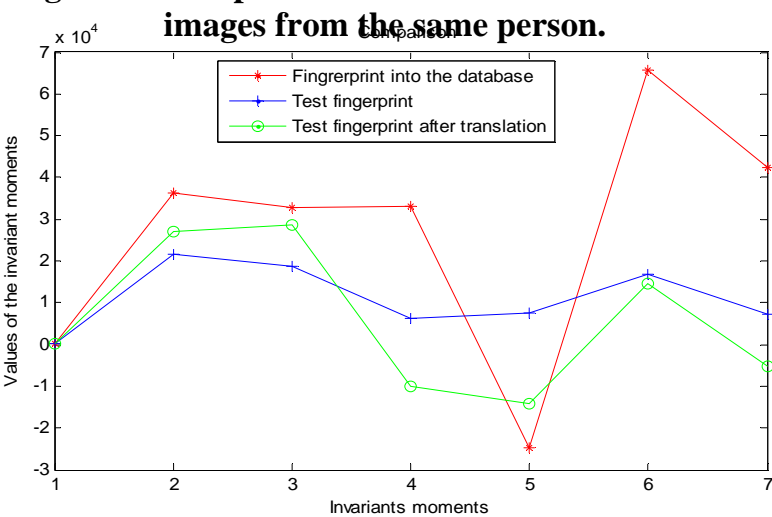

Figure 11. Graph of the invariant moments of two images from two different persons.

The first image Figure 10, is the graph obtained of the fingerprint images of the same person, the second image is the graph obtained of the fingerprint image from the two different persons Figure 11.

As it can be see, using invariant moments to generate the graph. The graph from the stored fingerprint is similar to the test image after the translation, when the fingerprint images are the same person Figure 10. However, when the fingerprint images are from different people, the graph generated by the invariant moments are different Figure 11.

The comparison was made using the feature vectors and different thresholds. In the comparison the distances and angles are duties to be equals, but the coordinates can be a few different, because at the moment when the fingerprint was capture, the finger had not the same position. After that the translation process is made and then compute of the invariants moments to rotation and translation was using the 10 by 10 window to obtain the values.

In table $\mathbf{1}$ are the results obtained in the recognition with verification stage:

\begin{tabular}{|l|c|c|c|}
\hline & Threshold & Window & Percentage \\
\hline Training & 10 & 10 & 98.46 \\
\hline $\begin{array}{l}\text { Recognitio } \\
\mathrm{n}\end{array}$ & 10 & 10 & 84.61 \\
\hline
\end{tabular}

Table 1. Final acceptance percentages.

\section{Conclusions}

Our proposal was, using two algorithms for image enhancement and also, the invariant moments in the verification phase. The percentage of the true acceptance can be increased to an acceptable value.

The percentage of the same minutiae in different images from the same person using both algorithms was 85.75, this is better than if we use only one process for the image enhancement: FFT that was 76.85 or Gabor that was 80.53. Our proposal to implement two algorithms for obtaining an enhancement image give us good results. If two fingerprints minutiae from the same person are compared it can be seen that the same fingerprint minutiae keep the same features although they do not have the same position at the time to computed the invariant moments.

The invariant moments algorithm gave us good results, when the test image was translated Figure 9, the graph of test image translated is very similar to the fingerprint stored. Then our proposal had a good performance because the true acceptance percentage was increased and the false acceptance was decreased.

\section{References}

[1] L. Hong, Y. Wang and A. K. Jain, "Fingerprint Image Enhancement: Algorithm and Performance Evaluation", Transactions on PAMI 21 (4) (1998) 777-789.

[2] L. O’Gormann and J.V.Nickerson, "An approach to fingerprint filter design”, Pattern Recognition 22 (1) (1989) 29-38.

[3] S. Greenberg, M. Aladjem, D. Kogan and I. Dimitrov, "Fingerprint image enhancement using filtering techniques", , in: International Conference on Pattern Recognition, Vol. 3, 2000, pp. 326-329.

[4] G. Z. Yang, P. Burger, D. N. Firmin and S. R Underwood, "Structure adaptive anisotropic image filtering”, Image and Vision Computing 14 (1996) 135-145.

[5] S. Qian and D. Chen, "Joint time-frequency analysis, methods and applications”, Prentice Hall, 1996. 
[6] B. G. Sherlock, D.M.Monro and K.Millard, "Fingerprint enhancement by directional Fourier filtering", in: Visual Image Signal Processing, Vol. 141, 1994, pp. 87-94.

[7] T. S. Lee, "Image representation using 2D gabor wavelets”, Transactions on PAMI 18 (10) (1996), 959-971.

[8] Ming-Kuei Hu,"Visual Pattern Recognition by Moment Invariants", IRE TRANSACTIONS ON INFORMATION THEORY, 1962. 